\title{
Chylothorax after blunt trauma
}

\author{
Megan Litzau, Julie Welch
}

Keywords: Chylothorax; Blunt trauma; Pleural effusion; Thoracic duct

A 66 yo female presented to the ED with 4 days of progressive shortness of breath. She was involved in a motor vehicle crash 7 days prior sustaining left rib fractures and non-operative cervical spine and pubic rami fractures. She was admitted for 3 days and managed conservatively. She denied fevers or hemoptysis. Physical exam findings included stable vital signs and diminished breath sounds over her right lung field. Chest X-ray and Chest CT revealed a large right pleural effusion (Images 1 and 2). Chest tube placement returned $>1 \mathrm{l}$ of milky pink fluid, consistent with a chylothorax (Image 3). Fluid analysis confirmed high triglyceride content. The patient was admitted to the trauma service with thoracic surgery consultation. She remained on a low fat diet for ten days until the chest tube output decreased to a serosanguinous consistency. On hospital day 9 her chest tube was removed, and she was discharged to home.

This is the author's manuscript of the article published in final edited form as:

Litzau, M., \& Welch, J. (2018). Chylothorax after blunt trauma. Visual Journal of Emergency Medicine, 10, 58-59. https://doi.org/10.1016/j.visj.2017.08.023 


\section{Figures}

Image 1. Chest x-ray revealing a large right-sided pleural effusion (chylothorax).

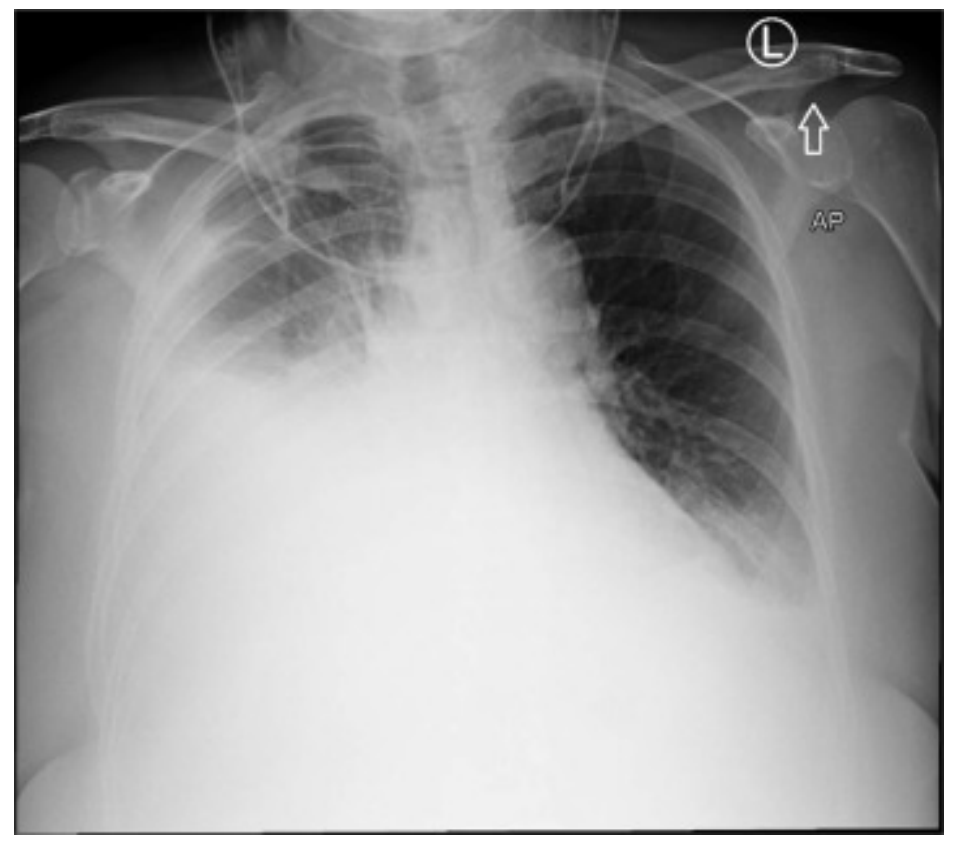

Image 2. Chest CT demonstrating a large right pleural effusion (chylothorax), dense consolidation of the right mid and lower lung zones.

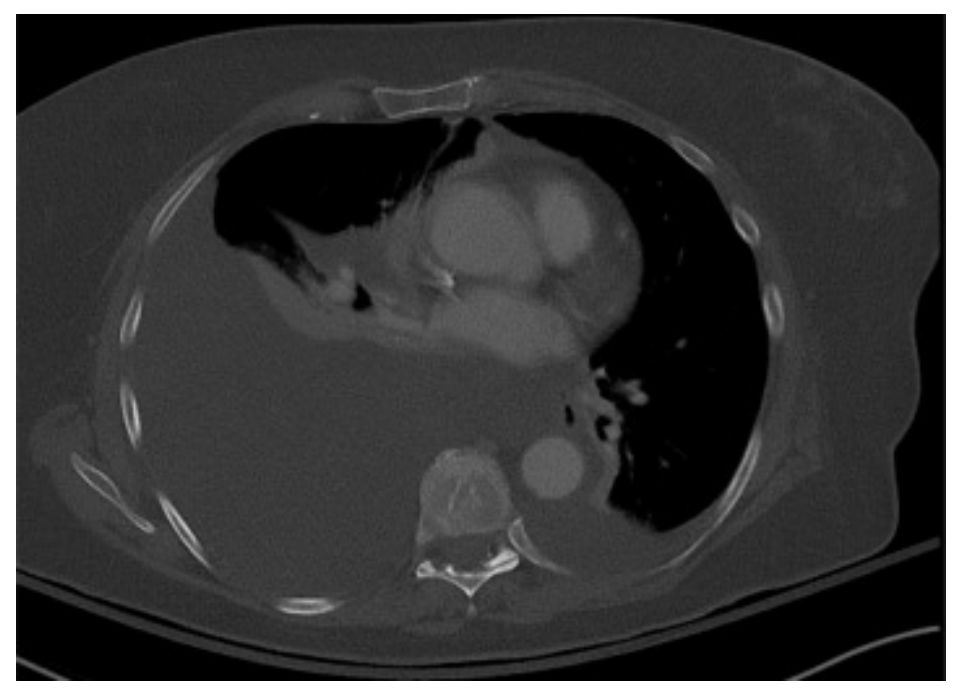


Image 3. Chest tube pleuravac with over $1 \mathrm{l}$ of milky pink fluid (high triglyceride content) consistent with chylothorax.

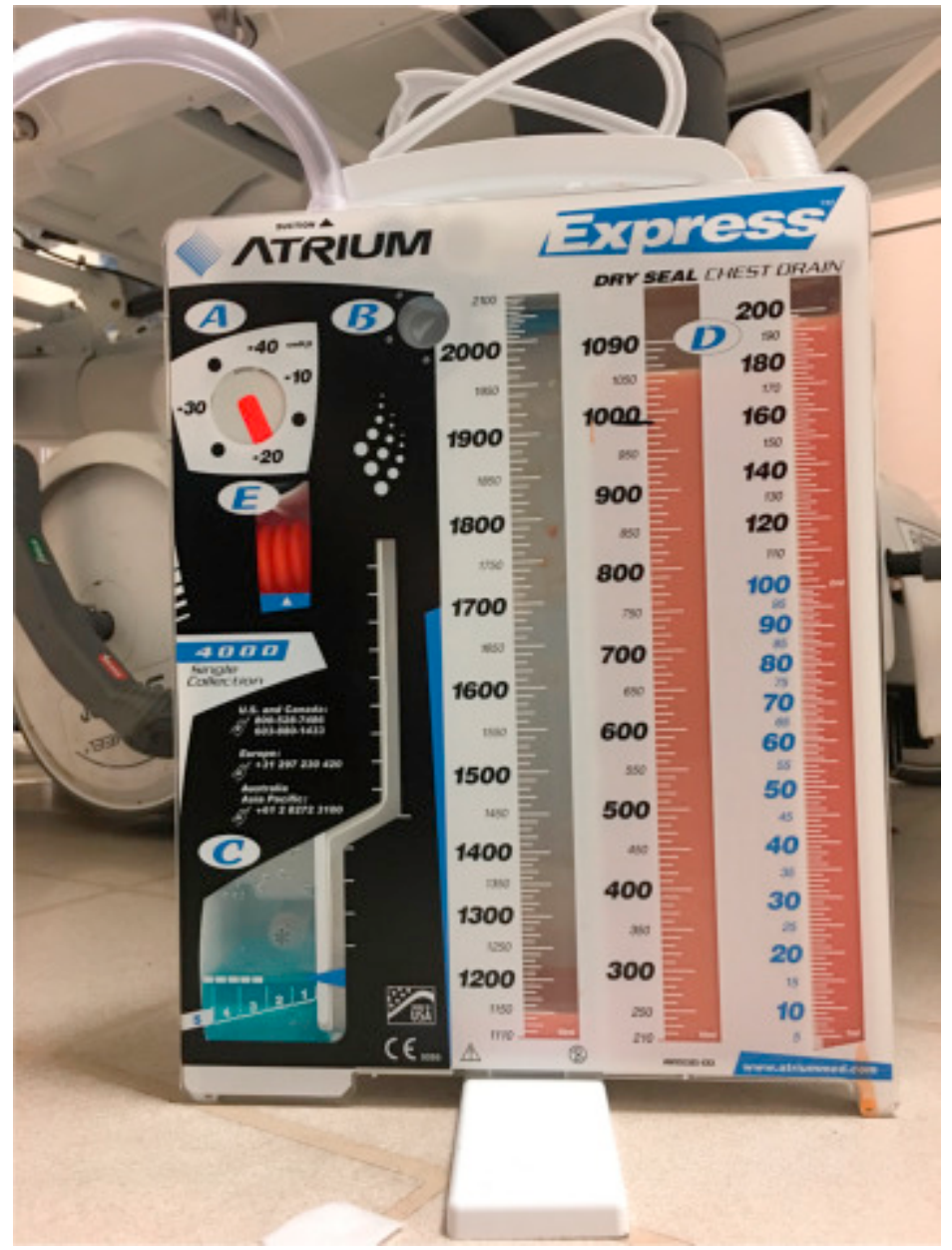




\section{Appendix A. Supplementary material}

\section{Further Reading}

1. Doerr CH, Allen MS, Nichols FC, Ryu JH. Etiology of chylothorax in 203 patients. Mayo Clin Proc. 2005;80(7):867-870.

2. Doerr CH, Miller DL, Ryu JH. Chylothorax. Semin Respir Crit Care Med. 2001;22(6):617-626.

3. Adams SD. Chylothorax. Medscape. Accessed August 72017.

(http://emedicine.medscape.com/article/172527). 


\section{Questions}

1. A 55 yo male presents with 3 days of dypnsea. Chest $x$-ray shows opacification of the left chest. A chest tube has milky output. As the chylothorax is on the left side, this indicates that the injury to the thoracic duct is above what level?
a. T3
b. T5
c. $\mathrm{T} 7$
d. T9
e. T11

2. A 30 yo male is admitted for rib fractures and orthopedic injuries. On day 7 he develops dyspnea. Chest $\mathrm{x}$-ray shows a large right effusion and chest tube output is milky white. Which of the following is a rare finding in chylothorax?
a. Chest pain
b. Shortness of breath (dyspnea)
c. Exercise intolerance
d. Chest heaviness
e. Fatigue

3. Which of the following represents the most common cause of chylothorax?
a. Penetrating trauma to the chest
b. Blunt trauma to the chest 
c. Iatrogenic trauma during thoracic surgery

d. Tuberculosis

e. Malignancy 


\section{Answers}

1. T5. Explanation: A chylothorax is a rare condition that results from a leak or tear in the thoracic duct which carries lymphatic fluid resulting in accumulation of chyle in the pleural space (effusion). The thoracic duct originates in the left thorax and crosses the mediastinum from left to right at the 5th thoracic vertebral body. As such, injury to the lymphatic system above this level will lead to left sided effusion while injury below this level will lead to right sided effusion.

2. Chest pain. Explanation: The lymphatic material in a chylothorax does not produce irritation of the chest wall. As such, it does not generally cause irritation of the pleura and therefore typically presents as a painless, progressive shortness of breath resulting in exercise intolerance, fatigue and general chest heaviness as the volume of the effusion increases.

3. Malignancy. Explanation: A chylothorax develops from damage to the thoracic duct causing a chlyle leak in the pleural space. The most common causes of chylothroax are non-traumatic, with hematologic malignancy (e.g., lymphoma) accounting for over 50\% of cases. Other non-traumatic causes are not uncommon and include congential, idiopathic, cirrhosis, sarcoidosis, amyloidosis, tuberculosis, and filariasis. Traumatic injury is the second leading cause (25\%) of chylothorax, with iatrogenic injury during thoracic surgery accounting for most cases. Accidental blunt or penetrating trauma (i.e., motor vehicle crash or stabbing) are very rare causes of chylothorax. 